Pure and Applied Mathematics Quarterly

Volume 2, Number 1

(Special Issue: In honor of

John H. Coates, Part 1 of 2)

$199-217,2006$

\title{
A Note on $L$-packets
}

\author{
James Arthur
}

\section{To John Coates on his sixtieth birthday}

1. Introduction. Suppose that $G$ is a connected reductive algebraic group over a field $F$ of characteristic 0 . For example, we could take $G$ to be the group $S L(n)$ of unimodular $(n \times n)$-matrices. We assume for a moment that $F$ is any local field. In other words, $F$ is a finite extension of either the archimedean field $\mathbb{R}$ or a nonarchimedean $p$-adic field $\mathbb{Q}_{p}$.

One of the fundamental problems of local harmonic analysis is to classify the set $\Pi(G)$ of equivalence classes of irreducible representations of $G(F)$. The problem separates naturally into two parts. The first is to establish the local Langlands correspondence. This conjecture of Langlands asserts that $\Pi(G)$ is a disjoint union of finite subsets $\Pi_{\phi}$, indexed by (equivalence classes of) Langlands parameters $\phi$ for $G$. The sets $\Pi_{\phi}$ are called $L$-packets, since their constituents could then be equipped with a common set of $L$-functions and $\varepsilon$-factors, by the constructions of $[\mathrm{T}, \S 3-4]$ and $[\mathrm{B}, \S 12]$. The second part of the problem is to classify the representations in a given packet $\Pi_{\phi}$. One would like to characterize them directly in terms of data attached to $\phi$.

For archimedean $F$, the classification was established in general by Langlands [La2], Knapp and Zuckerman [KZ], and Shelstad [S2]. For nonarchimedean $F$, the classification has been established in case $G=G L(n)$ by Harris and Taylor [HT] and Henniart $[\mathrm{H}]$. The packets $\Pi_{\phi}$ for $G L(n)$ each contain one element, so the second part of the problem is trivial in this case. For more general $p$-adic groups, the problem remains open. The purpose of this note, which is partly expository, is to describe a conjectural characterization of the $L$-packets $\Pi_{\phi}$ attached to a general $p$-adic group $G$. In particular, we shall give a conjectural formula for the number of elements in any such packet.

Received April 2, 2005. 
We assume from now on that the local field $F$ is nonarchimedean. A Langlands parameter for $G$ is an $L$-homomorphism

$$
\phi: L_{F} \longrightarrow{ }^{L} G,
$$

from the local Langlands group

$$
L_{F}=W_{F} \times S U(2)
$$

to the $L$-group

$$
{ }^{L} G=\widehat{G} \rtimes W_{F}
$$

of $G$, that is $G$-relevant. We recall that $W_{F}$ is the Weil group of $F$, that $\widehat{G}$ is the complex dual group of $G$, and that $W_{F}$ acts on $\widehat{G}$ through its projection onto a finite quotient of the absolute Galois group

$$
\Gamma=\operatorname{Gal}(\bar{F} / F) .
$$

An $L$-homomorphism in this context is continuous and semisimple (in the sense that projection of $\phi(w)$ onto $\widehat{G}$ is semisimple for every $w \in W_{F}$ ), and commutes with the two projections onto $W_{F}$. We say that $\phi$ is $G$-relevant if any parabolic subgroup ${ }^{L} P$ of ${ }^{L} G$ that contains the image of $\phi$ corresponds to a parabolic subgroup $P$ of $G$ that is defined over $F$. Let $\Phi(G)$ be the set of equivalence classes of Langlands parameters for $G$, taken with respect to the relation defined by $\widehat{G}$-conjugacy. The local Langlands conjecture asserts that $\Pi(G)$ is a disjoint union over $\phi \in \Phi(G)$ of finite packets $\Pi_{\phi}$.

Suppose that $\phi$ represents a class in $\Phi(G)$. The centralizer

$$
S_{\phi}=\operatorname{Cent}\left(\widehat{G}, \phi\left(L_{F}\right)\right)
$$

in $\widehat{G}$ of the image of $\phi$ is a complex reductive subgroup of $\widehat{G}$. If $\phi$ is replaced by another representative $\phi_{1}$ of its equivalence class in $\Phi(G)$, there is an isomorphism from the associated group $S_{\phi_{1}}$ onto $S_{\phi}$, which is uniquely determined up to conjugacy in $S_{\phi}$. We shall write

$$
S_{\phi, \mathrm{ad}}=S_{\phi} / Z(\widehat{G})^{\Gamma}
$$

where $Z(\widehat{G})^{\Gamma}$ is the group of invariants in the center $Z(\widehat{G})$ of $\widehat{G}$ under the action of the Galois group $\Gamma$. Then $S_{\phi, \text { ad }}$ is a reductive subgroup of the adjoint group $\widehat{G}_{\text {ad }}$ of $\widehat{G}$. Finally, we form the group

$$
\mathcal{S}_{\phi}=S_{\phi, \mathrm{ad}} / S_{\phi, \mathrm{ad}}^{0}=S_{\phi} / S_{\phi}^{0} Z(\widehat{G})^{\Gamma}
$$

of connected components of $S_{\phi, \text { ad }}$. This is a finite group that is determined up to inner automomorphism by the class of $\phi$ in $\Phi(G)$.

Let $\Phi_{\text {temp }}(G)$ be the subset of classes in $\Phi(G)$ represented by Langlands parameters $\phi$ whose image projects onto a relatively compact subset of $\widehat{G}$. These are the classes whose corresponding $L$-packets $\Pi_{\phi}$ should consist of representations 
that are tempered, in the sense that their characters are tempered distributions on $G(F)$. One can represent a general element in $\Phi(G)$ by a twist

$$
\phi_{z}(w)=\phi(w) z^{\operatorname{ord}(w)}, \quad w \in L_{F},
$$

where $\phi$ represents a class in $\Phi_{\text {temp }}(G), z$ is a suitable point in $S_{\phi}^{0}$, and $\operatorname{ord}(w)$ is the (additive) valuation of the element in $F^{*}$ onto which $w$ projects. Using the Langlands correspondence for split tori, one can identity $z$ with an unramified quasicharacter of a Levi subgroup of $G$ whose $L$-group contains the image of $\phi$. It can be used to deform the corresponding induced representations from which the packet $\Pi_{\phi}$ would be constructed. In fact, if $\phi$ is chosen suitably (relative to $\phi_{z}$ ), there should be a bijection $\pi \rightarrow \bar{\pi}_{z}$ from $\Pi_{\phi}$ to $\Pi_{\phi_{z}}$, obtained by taking Langlands quotients of corresponding deformations of tempered representations $\pi$ by $z$. A suitable characterization of the representations in $\Pi_{\phi}$ would therefore automatically yield a corresponding characterization of the representations in the packets $\Pi_{\phi_{z}}$. It would therefore be sufficient to consider the case that $\phi$ belongs to $\Phi_{\text {temp }}(G)$.

Suppose that $G$ is quasisplit, and that $\phi$ represents a class in $\Phi_{\text {temp }}(G)$. In this case, it is generally believed that there is a bijection

$$
\pi \longrightarrow\langle s, \pi\rangle, \quad s \in \mathcal{S}_{\phi}, \pi \in \Pi_{\phi},
$$

from $\Pi_{\phi}$ onto the set of irreducible characters of $\mathcal{S}_{\phi}$. This bijection would depend on normalizations of Langlands-Shelstad transfer factors, and would therefore not be completely canonical. However, it ought to be well defined up to the action of the group of one-dimensional characters of $\mathcal{S}_{\phi}$ on the set of irreducible characters. This bijection was established for $G=S L(2)$ in [LL]. (See also [S1, §11-12] and [La1, §IV.2].) Variants were established for real groups in [S2], and split $p$-adic groups in $[\mathrm{KL}]$.

Our goal is to formulate a generalization of this conjectural bijection. We shall state a general characterization of the packets $\Pi_{\phi}$ in $\S 3$ as a formal conjecture. I am not sure to what extent the conjecture is new. Variants were established for real groups in $[\mathrm{AV}]$ and adjoint $p$-adic groups in $[\mathrm{Lu}]$, and were stated for general $p$-adic groups in $[\mathrm{V}]$. The conjecture might simply be a reformulation of some of the constructions from $[\mathrm{V}]$. In any case, our emphasis will be somewhat different. We are going to focus on the transfer factors of Langlands and Shelstad. In particular, we shall use an equivariance property for automorphisms of endoscopic data, which can be derived from the definition [LS] of transfer factors, to illustrate how the general situation differs from the quasisplit case.

The equivariance property is in fact at the heart of the paper. It provides both motivation and evidence for the general conjecture. Its proof is not difficult, but will be given elsewhere in the interests of brevity. Its formal statement, formula (3.1) of $\S 3$, does require a short excursion into the theory of endoscopy. This 
makes the paper somewhat technical in places, despite its claim of being partly expository.

I would like to thank the referee for a number of thoughtful suggestions and remarks.

2. Transfer. Assume that $G$ is a general (connective, reductive) group over $F$. If $\phi$ represents a class in $\Phi_{\text {temp }}(G)$, the conjectural $L$-packet $\Pi_{\phi}$ should be defined in terms of Langlands-Shelstad transfer mappings. As correspondences of functions in Hecke algebras, these mappings remain conjectural. However, Waldspurger has shown that their existence is implied by the fundamental lemma [W1]. Recently, Laumon and Ngo [LN] and Waldspurger [W2] have established the fundamental lemma for unitary groups. The Langlands-Shelstad mappings therefore exist for any unitary group over $F$. We assume henceforth that they exist for our given $G$.

The transfer mappings are attached to endoscopic data $\left(G^{\prime}, \mathcal{G}^{\prime}, s^{\prime}, \xi^{\prime}\right)$ for $G$ [LS, (1.2)], which we often denote simply by the symbol $G^{\prime}$. Thus, $G^{\prime}$ can be regarded as a quasisplit group with some extra structure. The three extra components are a split extension $\mathcal{G}^{\prime}$ of $W_{F}$ by a dual group $\widehat{G}^{\prime}$ of $G^{\prime}$, an $L$-embedding $\xi^{\prime}$ of $\mathcal{G}^{\prime}$ into ${ }^{L} G$, and a semisimple element $s^{\prime} \in \widehat{G}$ whose connected centralizer equals the image $\xi^{\prime}\left(\widehat{G}^{\prime}\right)$ of $\widehat{G}^{\prime}$, and which we can also assume commutes with the image $\xi^{\prime}\left(\mathcal{G}^{\prime}\right)$ of $\mathcal{G}^{\prime}$ in ${ }^{L} G$.

Endoscopic data can be motivated in terms of Langlands parameters. Suppose that

$$
\phi^{\prime}: L_{F} \longrightarrow \mathcal{G}^{\prime}
$$

is an $L$-homomorphism associated with the endoscopic datum $G^{\prime}$. The composition $\phi=\xi^{\prime} \circ \phi^{\prime}$ is then an $L$-homomorphism from $L_{F}$ to ${ }^{L} G$. The point $s^{\prime}$ lies in the centralizer $S_{\phi}$, and its $Z(\widehat{G})^{\Gamma}$-coset $s$ is a semisimple element in the subgroup $S_{\phi, \text { ad }}$ of $\widehat{G}_{\text {ad }}$. Conversely, given any pair $(\phi, s)$, we can construct an associated endoscopic datum $\left(G^{\prime}, \mathcal{G}^{\prime}, s^{\prime}, \xi^{\prime}\right)$. We take $s^{\prime}$ to be any representative of $s$ in $S_{\phi}$, $\mathcal{G}^{\prime}$ to be the product

$$
\mathcal{G}^{\prime}=\widehat{G}^{\prime} \phi\left(L_{F}\right),
$$

where $\widehat{G}^{\prime}$ is the connected centralizer of $s^{\prime}$ in $\widehat{G}, \xi^{\prime}$ to be the identity $L$-embedding of $\mathcal{G}^{\prime}$ into ${ }^{L} G$, and $G^{\prime}$ to be any quasisplit group over $F$ for which $\widehat{G}^{\prime}$ is a dual group (relative to the $L$-action of $W_{F}$ on $\widehat{G}^{\prime}$ defined by $\mathcal{G}^{\prime}$ ). One could always compress the set of all endoscopic data by identifying $s^{\prime}$ with its $Z(\widehat{G})^{\Gamma}$-coset in $\widehat{G}, \mathcal{G}^{\prime}$ with its image $\xi^{\prime}\left(L_{F}\right)$ in $\widehat{G}, \xi^{\prime}$ with the identity $L$-embedding, and $G^{\prime}$ with its isomorphism class (with the extra structure that specifies the isomorphism between any two groups in the class is up to inner automorphisms). With this 
interpretation, the mapping

$$
\left(G^{\prime}, \phi^{\prime}\right) \longrightarrow(\phi, s)
$$

becomes a bijection. We will be interested in the restriction of the mapping to pairs $\left(G^{\prime}, \phi^{\prime}\right)$ that are $G$-relevant, in the sense that the corresponding $L$ homomorphism $\phi$ is $G$-relevant.

There is a broader equivalence relation on the set of pairs $\left(G^{\prime}, \phi^{\prime}\right)$, defined by the notion of isomorphism of endoscopic data [LS, (1.2)]. (We will review this notion at the beginning of the next section.) There is also an equivalence relation on the set of pairs $(\phi, s)$. It is defined by projecting $s$ to the quotient $\mathcal{S}_{\phi}$ of $S_{\phi, \text { ad }}$, and then letting $\widehat{G}$ act by conjugation. For example, the equivalence class of a $G$-relevant pair can be identified with (the class of) a Langlands parameter $\phi \in \Phi(G)$ and a conjugacy class $s$ in $\mathcal{S}_{\phi}$. It is not hard to show that the mapping (2.1) lifts to a surjective mapping between the two families of equivalence classes, with finite fibres. We say that $\left(G^{\prime}, \phi^{\prime}\right)$ is elliptic if the group $S_{\phi, \text { ad }}$ is finite, which is to say that it equals $\mathcal{S}_{\phi}$. A similar notion makes sense for pairs $(\phi, s)$. The mapping (2.1) restricts to a bijection between families of equivalence classes of elliptic pairs.

We return to the description of transfer mappings. The Langlands-Shelstad transfer mapping for a given endoscopic datum $G^{\prime}$ is defined in terms of a transfer factor $\Delta$. We recall that $\Delta$ depends on the choice of a suitable central extension

$$
1 \longrightarrow \widetilde{C}^{\prime} \longrightarrow \widetilde{G}^{\prime} \longrightarrow G^{\prime} \longrightarrow 1
$$

of $G^{\prime}$ over $F$, and a suitable $L$-embedding

$$
\widetilde{\xi}^{\prime}: \mathcal{G}^{\prime} \longrightarrow{ }^{L} \widetilde{G}^{\prime}
$$

It is a function $\Delta\left(\delta^{\prime}, \gamma\right)$ of a strongly $G$-regular, stable conjugacy class $\delta^{\prime}$ in $\widetilde{G}^{\prime}(F)$ and a strongly regular conjugacy class $\gamma$ in $G(F)$. Once $\widetilde{G}^{\prime}$ and $\widetilde{\xi}^{\prime}$ have been fixed, $\Delta$ is then determined up to a multiplicative constant of absolute value one. It is $\left(\widetilde{\eta}^{\prime}\right)^{-1}$-equivariant under translation of $\delta^{\prime}$ by $\widetilde{C}^{\prime}(F)$, where $\widetilde{\eta}^{\prime}$ is a character on $\widetilde{C}^{\prime}(F)$ determined by $\widetilde{\xi}^{\prime}$. The transfer mapping sends a function $f$ in the Hecke algebra $\mathcal{H}(G)$ on $G(F)$ to the function

$$
f^{\prime}\left(\delta^{\prime}\right)=f_{\Delta}^{\prime}\left(\delta^{\prime}\right)=\sum_{\gamma} \Delta\left(\delta^{\prime}, \gamma\right) f_{G}(\gamma)
$$

of $\delta^{\prime}$, where

$$
f_{G}(\gamma)=\left|D^{G}(\gamma)\right|^{\frac{1}{2}} \int_{G_{\gamma}(F) \backslash G(F)} f\left(x^{-1} \gamma x\right) \mathrm{d} x
$$

is the invariant orbital integral of $f$ at $\gamma$. Our assumption is that as $\delta^{\prime}$ varies, $f^{\prime}\left(\delta^{\prime}\right)$ is the stable orbital integral of some function in the equivariant Hecke algebra $\mathcal{H}\left(\widetilde{G}^{\prime}, \widetilde{\eta}^{\prime}\right)$ on $\widetilde{G}^{\prime}(F)$. 
We shall also need to grant one of the basic tenets of the conjectural theory of endoscopy. It is the existence of a natural stable character

$$
f \longrightarrow f^{G}(\phi), \quad f \in \mathcal{H}(G),
$$

for any Langlands parameter $\phi$. Our interest is not so much in this property for $G$, but rather in its analogue for the quasisplit groups $\widetilde{G}^{\prime}$ attached to $G$. This is what we assume. Suppose that $\phi^{\prime}$ is an $L$-homomorphism from $L_{F}$ to $\mathcal{G}^{\prime}$, as above. The composition

$$
\widetilde{\phi}^{\prime}=\widetilde{\xi}^{\prime} \circ \phi^{\prime}
$$

is then a Langlands parameter for $\widetilde{G}^{\prime}$, whose corresponding stable character could be integrated against the function $f^{\prime}\left(\delta^{\prime}\right)$ of $\delta^{\prime}$. This would provide an invariant distribution

$$
f \longrightarrow f^{\prime}\left(\phi^{\prime}\right), \quad f \in \mathcal{H}(G),
$$

on $G(F)$.

Indeed, the function $f^{\prime}\left(\delta^{\prime}\right)$ is $\left(\widetilde{\eta}^{\prime}\right)^{-1}$-equivariant. Both the stable character $\Theta\left(\widetilde{\phi^{\prime}}, \delta^{\prime}\right)$ of $\phi^{\prime}$, and its normalization

$$
\Phi\left(\widetilde{\phi}^{\prime}, \delta^{\prime}\right)=\left|D^{\tilde{G}^{\prime}}\left(\delta^{\prime}\right)\right|^{\frac{1}{2}} \Theta\left(\widetilde{\phi}^{\prime}, \delta^{\prime}\right)
$$

by the Weyl discriminant are supposed to be $\widetilde{\eta}^{\prime}$-equivariant under translation of $\delta^{\prime}$ by $\widetilde{C}^{\prime}(F)$. We can therefore define the distribution on $G(F)$ as an integral

$$
f^{\prime}\left(\phi^{\prime}\right)=f_{\Delta}^{\prime}\left(\phi^{\prime}\right)=\int \Phi\left(\widetilde{\phi}^{\prime}, \delta^{\prime}\right) f^{\prime}\left(\delta^{\prime}\right) \mathrm{d} \delta^{\prime},
$$

relative to a suitable measure on the space of strongly regular, stable conjugacy classes in $G^{\prime}(F)$. We would assume that $\Theta\left(\widetilde{\phi}^{\prime}, \cdot\right)$ is a finite linear combination of irreducible characters on $\widetilde{G}^{\prime}(F)$. The convergence of the integral is then a consequence of Harish-Chandra's theory of $p$-adic characters, and the general structure of the transfer factor $\Delta\left(\delta^{\prime}, \gamma\right)$. Defined in this way, the distribution does not actually depend on the Langlands-Shelstad transfer conjecture. However, it relies on the existence of a stable character attached to $\widetilde{\phi^{\prime}}$, which is undoubtedly deeper.

As the notation suggests, the distribution $f^{\prime}\left(\phi^{\prime}\right)$ depends on $\phi^{\prime}$ rather than $\widetilde{\phi^{\prime}}$. In other words, it is independent of the choice of $\widetilde{G}^{\prime}$ and $\widetilde{\xi}^{\prime}$. A variation in the choice of $\widetilde{\xi}^{\prime}$, for example, is compensated by a corresponding variation in the composition $\widetilde{\phi}^{\prime}=\widetilde{\xi}^{\prime} \circ \phi^{\prime}$. However, $f^{\prime}\left(\phi^{\prime}\right)$ does still depend on the choice of transfer factor $\Delta$. We can think of $\Delta$ as a family of transfer factors, one for each choice of $\left(\widetilde{G}^{\prime}, \widetilde{\xi}^{\prime}\right)$, which satisfy natural compatibility conditions as these choices vary. This object, which we can call a transfer family for $G^{\prime}$, is uniquely determined up to a multiplicative constant of absolute value one. The same is therefore true of $f^{\prime}\left(\phi^{\prime}\right)$. 
Given what we have assumed, the distribution $f \rightarrow f^{\prime}\left(\phi^{\prime}\right)$ on $G(F)$ can be seen to be a linear combination

$$
f^{\prime}\left(\phi^{\prime}\right)=\sum_{\pi} \Delta\left(\phi^{\prime}, \pi\right) f_{G}(\pi), \quad f \in \mathcal{H}(G),
$$

of irreducible characters. The linear form

$$
f_{G}(\pi)=\int \Phi(\pi, \gamma) f_{G}(\gamma) \mathrm{d} \gamma
$$

here is the character of the irreducible representation $\pi \in \Pi(G)$, while the coefficient $\Delta\left(\phi^{\prime}, \pi\right)$ is a complex number that depends in the obvious way on the choice of transfer family $\Delta$. As we have noted, the composition $\phi=\xi^{\prime} \circ \phi^{\prime}$ is a Langlands parameter for $G$. If $\phi$ is any $G$-relevant Langlands parameter, the $L$-packet $\Pi_{\phi}$ could be defined as the set of $\pi$ for which the coefficient $\Delta\left(\phi^{\prime}, \pi\right)$ does not vanish for some pair $\left(G^{\prime}, \phi^{\prime}\right)$ that maps to $\phi$. To establish the local Langlands classification, one would need to show that the packets $\Pi_{\phi}$ are finite, disjoint, and have union equal to $\Pi(G)$.

Our concern here is in the supplementary question of how to characterize the representations in a given packet $\Pi_{\phi}$. We shall recall the precise form of the conjectural parametrization in case $G$ is quasisplit over $F$.

Assume that $G$ is quasisplit, that $\phi$ is a fixed Langlands parameter for $G$, and that $s$ is a semisimple element in $S_{\phi, \text { ad }}$. Let $\left(G^{\prime}, \phi^{\prime}\right)$ be the pair that maps to $(\phi, s)$, in the sense above. It is then not hard to show that the linear form

$$
f^{\prime}\left(\phi^{\prime}\right), \quad f \in \mathcal{H}(G),
$$

depends only on the isomorphism class of $\left(G^{\prime}, \phi^{\prime}\right)$ (defined by isomorphism of endoscopic data). Implicit in this assertion is the fact that the transfer family $\Delta$ for $G^{\prime}$ relative to which $f^{\prime}\left(\phi^{\prime}\right)$ is defined has a canonical matching transfer family for any endoscopic datum isomorphic to $G^{\prime}$. (See [A2].) In fact, $f^{\prime}\left(\phi^{\prime}\right)$ can be seen to depend only on the image $(\phi, s)$ of $\left(G^{\prime}, \phi^{\prime}\right)$ under the mapping above. The upshot is that we can regard $f^{\prime}\left(\phi^{\prime}\right)$ as an invariant of the $\widehat{G}$-orbit of $(\phi, s)$ (and an element $\Delta$ in the $U(1)$-torsor of matching transfer families). The same is therefore true of the coefficients $\Delta\left(\phi^{\prime}, \pi\right)$.

Assume now that $\phi$ is tempered, in that it represents a class in $\Phi_{\text {temp }}(G)$. The conjectural parametrization of the representations in the packet $\Pi_{\phi}$ is as follows. There should be a $U(1)$-valued function

$$
\rho(\Delta, s), \quad s \in S_{\phi, \text { ad }},
$$

with the following properties:

(i) $\rho(t \Delta, s)=t \rho(\Delta, s)$, $t \in U(1)$. 
(ii) For any representation $\pi \in \Pi_{\phi}$, the function

$$
\langle s, \pi\rangle=\rho(\Delta, s)^{-1} \Delta\left(\phi^{\prime}, \pi\right),
$$$$
s \in S_{\phi, \mathrm{ad}},
$$

depends only on the image of $s$ in $\mathcal{S}_{\phi}$, and lifts to an irreducible character on $\mathcal{S}_{\phi}$.

(iii) The mapping

$$
\pi \longrightarrow\langle\cdot, \pi\rangle, \quad \pi \in \Pi_{\phi},
$$

is a bijection from $\Pi_{\phi}$ onto the set of irreducible characters on $\mathcal{S}_{\phi}$.

In particular the number of representations in $\Pi_{\phi}$ should be equal to the number of conjugacy classes in $\mathcal{S}_{\phi}$. The function $\rho(\Delta, s)$ would not be unique. However, it would clearly be uniquely determined up to multiplication by a one dimensional character on $\mathcal{S}_{\phi}$.

The conjectural characterization of the packet $\Pi_{\phi}$ we have just described for quasisplit $G$ seems to be part of accepted lore. However, fails in general. For example, it does not hold if $G$ is the subgroup of elements of norm 1 in a quaternion algebra over $F$. In this case, Labesse and Langlands [LL] describe parameters $\phi$ for $G$ such that the pairing $\langle s, \pi\rangle$, defined for any function $\rho(\Delta, s)$ as above, fails to be a character on $\mathcal{S}_{\phi}$. This failure turns out to be related to the behaviour of the transfer mapping $f \rightarrow f^{\prime}$ under automorphisms.

3. A conjecture. We return to the case that $G$ is an arbitrary connected reductive group over the local $p$-adic field $F$. The transfer factors then depend implicitly on one other choice, that of a quasisplit inner twist for $G$. This is an isomorphism

$$
\psi: G \longrightarrow G^{*}
$$

from $G$ to a quasisplit group $G^{*}$ over $\bar{F}$ such that for every element $\sigma$ in the Galois group $\Gamma=\operatorname{Gal}(\bar{F} / F)$, the automorphism $\psi \circ \sigma(\psi)^{-1}$ of $G^{*}$ is inner. In case $G$ itself is quasisplit, one usually takes $\psi$ to be the identity mapping, so the implicit dependence on a choice of $\psi$ is not relevant to the discussion at the end of the last section. In general, we write

$$
\widehat{Z}_{\mathrm{sc}}=Z\left(\widehat{G}_{\mathrm{sc}}^{*}\right)
$$

for the center of $\widehat{G}_{\mathrm{sc}}^{*}$, the simply connected cover of the derived group of $\widehat{G}^{*}$.

There is a remarkable classification of inner twists, which relies ultimately on local class field theory. It takes the form of a canonical bijection

$$
(G, \psi) \longrightarrow \widehat{\zeta}_{G}
$$


from the set of isomorphism classes of pairs $(G, \psi)$ attached to a given $G^{*}$ onto the group of characters $\widehat{\zeta}_{G}$ on the finite group $\widehat{Z}_{\mathrm{sc}}^{\Gamma}$ of $\Gamma$-invariants in $\widehat{Z}_{\mathrm{sc}}$. (See [K, Theorem 1.2]. The first set is bijective with the Galois cohomology set $H^{1}\left(F, G_{\mathrm{ad}}^{*}\right)$, essentially by definition. This set in turn is mapped bijectively onto the group of characters on $\widehat{Z}_{\mathrm{sc}}^{\Gamma}$ by the appropriate duality theorem.)

We use $\psi$ to identify $\widehat{G}^{*}$ with the dual group $\widehat{G}$ of $G$. In particular, we identify $\widehat{Z}_{\text {sc }}$ with the center $Z\left(\widehat{G}_{\mathrm{sc}}\right)$ of the simply connected cover of the derived group of $\widehat{G}$. We are interested in the character $\widehat{\zeta}_{G}$ on $\widehat{Z}_{\mathrm{sc}}^{\Gamma}$ attached to the pair $(G, \psi)$.

The notion of an isomorphism of endoscopic data, mentioned in $\S 2$, is central to our discussion. We should therefore review the definition from [LS, (1.2)], where it was introduced only in the form of an equivalence relation on endoscopic data. An isomorphism from $\left(G^{\prime}, \mathcal{G}^{\prime}, s^{\prime}, \zeta^{\prime}\right)$ to $\left(G_{1}^{\prime}, \mathcal{G}_{1}^{\prime}, s_{1}^{\prime}, \xi_{1}^{\prime}\right)$ is an $F$-isomorphism $\alpha: G^{\prime} \rightarrow G_{1}^{\prime}$ that is dual to an isomorphism induced by conjugation by an element in $\widehat{G}$. More precisely, $\alpha$ comes with the supplementary data of an $L$-isomorphism $\beta: \mathcal{G}_{1}^{\prime} \rightarrow \mathcal{G}^{\prime}$ and an element $g \in \widehat{G}$ that satisfy natural compatibility conditions. Namely, the isomorphisms

$$
\Psi\left(G^{\prime}\right) \longrightarrow \Psi\left(G_{1}^{\prime}\right)
$$

and

$$
\Psi\left(\widehat{G}_{1}^{\prime}\right) \longrightarrow \Psi\left(\widehat{G}^{\prime}\right)
$$

of canonical based root data induced by $\alpha$ and $\beta$ are dual, the embeddings $\xi^{\prime}$ and $\xi_{1}^{\prime}$ are related by

$$
\operatorname{Int}(g) \cdot \xi^{\prime} \cdot \beta=\xi_{1}^{\prime},
$$

and the points $s^{\prime}$ and $s_{1}^{\prime}$ are such that $g s^{\prime} g^{-1}$ belongs to $Z(\widehat{G}) Z\left(\xi_{1}^{\prime}\right)^{0} s_{1}^{\prime}$, where $Z\left(\xi_{1}^{\prime}\right)$ is the centralizer in $\widehat{G}$ of the image $\xi_{1}^{\prime}\left(\mathcal{G}_{1}^{\prime}\right)$.

Suppose that $\alpha$ is an isomorphism between endoscopic data $G^{\prime}$ and $G_{1}^{\prime}$, as above. If $\phi^{\prime}$ is an $L$-homomorphism from $L_{F}$ to $\mathcal{G}^{\prime}$, the function

$$
\alpha \phi^{\prime}=\beta^{-1} \circ \phi^{\prime}
$$

is an $L$-homomorphism from $L_{F}$ to $\mathcal{G}_{1}^{\prime}$. It is the correspondence

$$
\left(G^{\prime}, \phi^{\prime}\right) \longrightarrow\left(\alpha G^{\prime}, \alpha \phi^{\prime}\right)
$$

obtained by varying $\alpha$ that provides the isomorphism class of pairs $\left(G^{\prime}, \phi^{\prime}\right)$ mentioned in $\S 2$. If $\Delta$ is a transfer factor for $G^{\prime}$ (with an auxiliary datum $\left(\widetilde{G}^{\prime}, \widetilde{\xi}^{\prime}\right)$ ), the function

$$
(\alpha \Delta)\left(\delta_{1}^{\prime}, \gamma\right)=\Delta\left(\alpha^{-1} \delta_{1}^{\prime}, \gamma\right)
$$

is a transfer factor for $G_{1}^{\prime}$ (with a corresponding auxiliary datum $\left(\widetilde{G}_{1}^{\prime}, \widetilde{\xi}_{1}^{\prime}\right)$ and a suitable extension of $\alpha$ from $\widetilde{G}^{\prime}$ to $\left.\widetilde{G}_{1}^{\prime}\right)$. In fact, if $\Delta$ represents a transfer family for 
$G^{\prime}, \alpha \Delta$ represents a transfer family for $G_{1}^{\prime}$. Suppose that the $L$-homomorphism $\phi^{\prime}$ above is $G$-relevant. It is then a consequence of the definitions that

$$
f_{\alpha \Delta}^{\prime}\left(\alpha \phi^{\prime}\right)=f_{\Delta}^{\prime}\left(\phi^{\prime}\right)=f^{\prime}\left(\phi^{\prime}\right), \quad f \in \mathcal{H}(G)
$$

We write $\operatorname{Aut}_{G}\left(G^{\prime}\right)$ for the group of automorphisms of the endoscopic datum $G^{\prime}$, and $\operatorname{Int}_{G}\left(G^{\prime}\right)$ for the subgroup of elements in $\operatorname{Aut}_{G}\left(G^{\prime}\right)$ for which the $F$-rational automorphism $\alpha$ of $G^{\prime}$ is inner. The quotient

$$
\operatorname{Out}_{G}\left(G^{\prime}\right)=\operatorname{Aut}_{G}\left(G^{\prime}\right) / \operatorname{Int}_{G}\left(G^{\prime}\right)
$$

is then a finite group of $F$-outer automorphisms $\alpha$ of the group $G^{\prime}$. Any $\alpha$ in this group comes with an element $g=g_{\alpha}^{\prime}$ in $\widehat{G}$ that is uniquely determined modulo the connected subgroup $\xi^{\prime}\left(\widehat{G}^{\prime}\right)$ of $\widehat{G}$. Given $G^{\prime}$ (as an endoscopic datum), let $\widetilde{s}$ be a point in the preimage in $\widehat{G}_{\text {sc }}$ of the image $s \in \widehat{G}_{\text {ad }}$ of the point $s^{\prime} \in \widehat{G}^{\prime}$ attached to $G^{\prime}$. For any given $\alpha \in \operatorname{Out}_{G}\left(G^{\prime}\right)$, let $\widetilde{g}_{\alpha}$ be any point in $\widehat{G}_{\mathrm{sc}}$ that projects to the image of $g_{\alpha}^{\prime}$ in $\widehat{G}_{\text {ad }}$. It is then easy to see that

$$
\widetilde{g}_{\alpha} \widetilde{s} \widetilde{g}_{\alpha}^{-1}=\widetilde{s} z(\alpha),
$$

for some point $z(\alpha)$ in $\widehat{Z}_{\mathrm{sc}}^{\Gamma}$, and that

$$
\alpha \longrightarrow z(\alpha), \quad \alpha \in \operatorname{Out}_{G}\left(G^{\prime}\right),
$$

is an injective homomorphism from $\operatorname{Out}_{G}\left(G^{\prime}\right)$ to $\widehat{Z}_{\mathrm{sc}}^{\Gamma}$. (See [A2].)

The character

$$
\alpha \longrightarrow \widehat{\zeta}_{G}(z(\alpha)), \quad \alpha \in \operatorname{Out}_{G}\left(G^{\prime}\right),
$$

on $\operatorname{Out}_{G}\left(G^{\prime}\right)$ obtained from $\widehat{\zeta}_{G}$ describes the behaviour of transfer under automorphisms. Suppose that $\Delta$ is a transfer family for $G^{\prime}$. Then for any $\alpha \in \operatorname{Out}_{G}\left(G^{\prime}\right)$, $\alpha \Delta$ is also a transfer family for $G^{\prime}$. The point we wish to emphasize is that $\alpha \Delta$ may not be equal to $\Delta$. One shows in fact that

$$
\alpha \Delta=\widehat{\zeta}_{G}(z(\alpha)) \Delta, \quad \alpha \in \operatorname{Out}_{G}\left(G^{\prime}\right) .
$$

(See $[\mathrm{A} 2, \S 2]$.$) This means that transfer families cannot be assigned in a consistent$ way to the endoscopic data in a given isomorphism class. One could summarize the situation by saying that the principal $U(1)$-bundle of transfer families over the space of endoscopic data in a given isomorphism class need not have a section. (This point was overlooked in several of the papers leading up to the stable trace formula [A1]. The necessary modifications are minor, and will appear elsewhere.)

In particular, suppose that

$$
\phi: L_{F} \longrightarrow{ }^{L} G
$$

is a Langlands parameter for $G$. If $s$ belongs to $S_{\phi, \text { ad }}$, there is a pair $\left(G^{\prime}, \phi^{\prime}\right)$ that maps to $(\phi, s)$. This pair is unique if we compress endoscopic data according to the innocuous convention in $\S 2$. Let $\mathcal{T}(\phi, s)$ be the $U(1)$-torsor of transfer families attached to $G$ and $G^{\prime}$. We can regard $\mathcal{T}(\phi, s)$ as the fibre of a principal 
$U(1)$-bundle on the $\widehat{G}$-orbit of the pair $(\phi, s)[\mathrm{A} 2]$. The point is that this bundle may not have a continuous section. We get around the problem by introducing an extension of the group $S_{\phi, \text { ad }}$.

Given $\phi$, we define $S_{\phi, \mathrm{sc}}$ to be the preimage of $S_{\phi, \text { ad }}$ in $\widehat{G}_{\mathrm{sc}}$. This group can also be characterized directly in terms of the image $\phi\left(L_{F}\right)$ of $L_{F}$ in ${ }^{L} G$. The points in $\phi\left(L_{F}\right)$ act by conjugation on the group $\widehat{G}_{\mathrm{sc}}$. It follows from the definitions that $S_{\phi, \text { sc }}$ is the subgroup of points $\widetilde{s} \in \widehat{G}_{\mathrm{sc}}$ such that for every $\lambda \in \phi\left(L_{F}\right)$, the commutator

is a point in $\widehat{Z}_{\mathrm{sc}}$ of the form

$$
z(\widetilde{s}, \lambda)=\lambda \widetilde{s} \lambda^{-1} \widetilde{s}^{-1}
$$

$$
z_{\lambda} \lambda(z) z^{-1}
$$

where $z \in \widehat{Z}_{\text {sc }}$ is independent of $\lambda, \lambda$ acts on $z$ through its projection onto the Galois group $\Gamma$, and $z_{\lambda}$ lies in the kernel of the mapping of $\widehat{Z}_{\mathrm{sc}}$ into $Z(\widehat{G})$. It is clear that $S_{\phi, \mathrm{sc}}$ is an extension of $S_{\phi, \text { ad }}$ by the center $\widehat{Z}_{\mathrm{sc}}$. We set

$$
\widetilde{\mathcal{S}}_{\phi}=S_{\phi, \mathrm{sc}} / S_{\phi, \mathrm{sc}}^{0} \text {. }
$$

Then $\widetilde{\mathcal{S}}_{\phi}$ is an extension of $\mathcal{S}_{\phi}$ by the quotient

$$
\widetilde{\mathcal{Z}}_{\phi}=\widehat{Z}_{\mathrm{sc}} / \widehat{Z}_{\mathrm{sc}}^{0}
$$

where

$$
\widehat{Z}_{\mathrm{sc}}^{0}=\widehat{Z}_{\mathrm{sc}} \cap S_{\phi, \mathrm{sc}}^{0} .
$$

The group $\widehat{Z}_{\mathrm{sc}}$ thus acts transitively on the fibres of the projection $\widetilde{s} \rightarrow s$ from $\widetilde{\mathcal{S}}_{\phi}$ to $\mathcal{S}_{\phi}$. It therefore acts transitively on the fibres of the projection

$$
\Gamma\left(\widetilde{\mathcal{S}}_{\phi}\right) \longrightarrow \Gamma\left(\mathcal{S}_{\phi}\right),
$$

where $\Gamma\left(\widetilde{\mathcal{S}}_{\phi}\right)$ and $\Gamma\left(\mathcal{S}_{\phi}\right)$ denote the sets of conjugacy classes in $\widetilde{\mathcal{S}}_{\phi}$ and $\mathcal{S}_{\phi}$ respectively. We define $\Gamma\left(\mathcal{S}_{\phi}, \widehat{\zeta}_{G}\right)$ to be the image in $\Gamma\left(\mathcal{S}_{\phi}\right)$ of those classes in $\Gamma\left(\widetilde{\mathcal{S}}_{\phi}\right)$ whose stabilizer in $\widehat{Z}_{\mathrm{sc}}$ is contained in the subgroup of $\widehat{Z}_{\mathrm{sc}}^{\Gamma}$ on which the character $\widehat{\zeta}_{G}$ equals 1. Then $\Gamma\left(\mathcal{S}_{\phi}, \widehat{\zeta}_{G}\right)$ is a subset of $\Gamma\left(\mathcal{S}_{\phi}\right)$ that is in general proper. I do not know whether it is always nonempty.

Conjecture. Suppose that $\phi: L_{F} \rightarrow{ }^{L} G$ is a tempered Langlands parameter for $G$.

(a) There is a $U(1)$-valued function

$$
\rho(\Delta, \widetilde{s}),
$$$$
\Delta \in \mathcal{T}(\phi, s), \widetilde{s} \in S_{\phi, \mathrm{sc}},
$$

with the following properties:
(i) $\rho(t \Delta, z \widetilde{s})=t \rho(\Delta, \widetilde{s}) \widehat{\zeta}_{\rho}(z)^{-1}$,
$t \in U(1), z \in \widehat{Z}_{\mathrm{sc}}$, 
where $\widehat{\zeta}_{\rho}$ is a fixed character on $\widehat{Z}_{\mathrm{sc}}$ whose restriction to $\widehat{Z}_{\mathrm{sc}}^{\Gamma}$ equals $\widehat{\zeta}_{G}$.

(ii) For any representation $\pi \in \Pi_{\phi}$, the function

$$
\langle\widetilde{s}, \pi\rangle=\rho(\Delta, \widetilde{s})^{-1} \Delta\left(\phi^{\prime}, \pi\right), \quad \widetilde{s} \in S_{\phi, \mathrm{sc}},
$$

depends only on the image of $\widetilde{s}$ in $\widetilde{\mathcal{S}}_{\phi}$, and descents to an irreducible character on $\widetilde{\mathcal{S}}_{\phi}$.

(iii) The mapping

$$
\pi \longrightarrow\langle\cdot, \pi\rangle, \quad \pi \in \Pi_{\phi},
$$

is a bijection from $\Pi_{\phi}$ onto the set of irreducible characters on $\widetilde{\mathcal{S}}_{\phi}$ whose central character on $\widehat{Z}_{\mathrm{sc}}$ equals $\widehat{\zeta}_{\rho}$.

(b) The number of representations in $\Pi_{\phi}$ equals the number of classes in the subset $\Gamma\left(\mathcal{S}_{\phi}, \widehat{\zeta}_{G}\right)$ of $\Gamma\left(\mathcal{S}_{\phi}\right)$.

Remarks. 1. We reiterate that we have had to make two assumptions in order even to state the conjecture. They are the existence of the transfer mapping $f \rightarrow f^{\prime}$, and the existence of the stable distribution on $\widetilde{G}^{\prime}(F)$ attached to $\widetilde{\phi}^{\prime}$. With these assumptions, the left hand side of (2.2) is defined, and so therefore are the coefficients $\Delta\left(\phi^{\prime}, \pi\right)$ in the expansion on the right hand side. The coefficients of course intervene in the property (a)(ii). They are an essential part of the conjecture.

2. The equivariance formula (3.1) is another essential ingredient, even though it is only implicit. Its proof in [A2] will be accompanied by more detailed discussion of some of the formal properties of endoscopy we have introduced here. At the end of the next section we shall try to show by example that (3.1) is at least suggestive of the conjecture.

3. The character $\widehat{\zeta}_{\rho}$ in (a)(i) might seem odd at first glance, insofar as it is defined on a group $\widehat{Z}_{\mathrm{sc}}$ that properly contains $\widehat{Z}_{\mathrm{sc}}^{\Gamma}$ in general. We shall discuss its meaning at the beginning of the next section.

4. The quotient $\langle\widetilde{s}, \pi\rangle$ is independent of the transfer family $\Delta$. Since it is assumed also to depend only on the conjugacy class of $\widetilde{s}$, it can be regarded as an invariant of the $\widehat{G}$-orbit of $(\phi, \widetilde{s})$. The normalizing factor $\rho(\Delta, \widetilde{s})$ in the definition of $\langle\widetilde{s}, \pi\rangle$ can be interpreted as a $\widehat{G}$-invariant section of the $U(1)$-bundle of transfer families over the $\widehat{G}$-orbit of $(\phi, \widetilde{s})$.

5. The function $\rho(\Delta, \widetilde{s})$ will not be unique. It seems reasonable to expect it to be well defined up to multiplication by a one-dimensional character on $\widetilde{\mathcal{S}}_{\phi}$ that is trivial on the image of $\widehat{Z}_{\mathrm{sc}}$. However, I have not been able to show that this is so. 
6. If the center of $G$ is connected, the derived group of $\widehat{G}$ is simply connected, and $\widehat{Z}_{\text {sc }}$ is contained in the center of $Z(\widehat{G})$. In this case, the conjecture is closely related to the discussion of [LS, (4.2)]. This was pointed out by Kottwitz, following earlier observations of Vogan.

7. If $G$ is an adjoint group, a parametrization of certain packets $\Pi_{\phi}$ was established in [Lu]. In case $G$ is an inner form of the adjoint split group $G^{*}=$ $S O(2 n+1)$, Moeglin and Waldspurger $[\mathrm{MW}]$ have shown that the parametrization in $[\mathrm{Lu}]$ coincides with that of the conjecture above.

8. Analogues of the conjecture were proved for real groups in $[\mathrm{AV}]$, and were stated for $p$-adic groups in $[\mathrm{V}]$. However, transfer factors do not appear to have an explicit role in these papers. It would be an interesting exercise to relate the Langlands-Shelstad transfer factors for real groups in [LS] directly to geometric constructions in $[\mathrm{AV}]$ and $[\mathrm{ABV}]$. Perhaps this would suggest geometric interpretations for the various components of the more complicated $p$-adic transfer factors.

4. Observations. We shall add some further observations, which are of an entirely elementary nature. The character $\widehat{\zeta}_{G}$ that determines $G$ as an inner twist is defined on the subgroup $\widehat{Z}_{\mathrm{sc}}^{\Gamma}$ of $\widehat{Z}_{\mathrm{sc}}$. The functions $\langle\widetilde{s}, \pi\rangle$ attached to $\Pi_{\phi}$ are supposed to have fixed central character $\widehat{\zeta}_{\rho}$ on $\widehat{Z}_{\mathrm{sc}}$. We are thus proposing an extension of $\widehat{\zeta}_{G}$ to a character on the larger group $\widehat{Z}_{\mathrm{sc}}$. What is its role?

Consider the canonical mapping of $\widehat{Z}_{\mathrm{sc}}$ into the abelianization

$$
\widetilde{\mathcal{S}}_{\phi, \mathrm{ab}}=\widetilde{\mathcal{S}}_{\phi} / \widetilde{\mathcal{S}}_{\phi, \mathrm{der}} \cong S_{\phi, \mathrm{sc}} / S_{\phi, \mathrm{sc}, \mathrm{der}} \cdot S_{\phi, \mathrm{sc}}^{0}
$$

of $\widetilde{\mathcal{S}}_{\phi}$. We claim that the kernel

$$
\widehat{Z}_{\phi, \mathrm{sc}, \mathrm{der}}=\widehat{Z}_{\mathrm{sc}} \cap\left(S_{\phi, \mathrm{sc}, \mathrm{der}} \cdot S_{\phi, \mathrm{sc}}^{0}\right)
$$

of this mapping is contained in the subgroup $\widehat{Z}_{\mathrm{sc}}^{\Gamma}$ of $\widehat{Z}_{\mathrm{sc}}$. To see this, suppose first that $\widetilde{s}_{1}$ is any element in the derived group $S_{\phi, \mathrm{sc}, \mathrm{der}}$ of $S_{\phi, \mathrm{sc}}$. Then $\widetilde{s}_{1}$ is a product of commutators

$$
\widetilde{t} \widetilde{u} \widetilde{t}^{-1} \widetilde{u}^{-1}, \quad \widetilde{t}, \widetilde{u} \in S_{\phi, \mathrm{sc}} .
$$

If $\lambda$ belongs to the image $\phi\left(L_{F}\right)$ of $L_{F}$ in ${ }^{L} G$, then $\lambda \widetilde{s} \lambda^{-1}$ is a corresponding product of commutators

$$
\begin{aligned}
\lambda \widetilde{t} \widetilde{u} \widetilde{t}^{-1} \widetilde{u}^{-1} \lambda^{-1} & =\left(\lambda \widetilde{t} \lambda^{-1}\right)\left(\lambda \widetilde{u} \lambda^{-1}\right)\left(\lambda \widetilde{t} \lambda^{-1}\right)^{-1}\left(\lambda \widetilde{u} \lambda^{-1}\right)^{-1} \\
& =(\widetilde{t z}(\widetilde{t}, \lambda))(\widetilde{u} z(\widetilde{u}, \lambda))(\widetilde{t z}(\widetilde{t}, \lambda))^{-1}(\widetilde{u} z(\widetilde{u}, \lambda))^{-1} \\
& =\widetilde{t} \widetilde{u} \widetilde{t}^{-1} \widetilde{u}^{-1},
\end{aligned}
$$

for points $z(\widetilde{t}, \lambda)$ and $z(\widetilde{u}, \lambda)$ in $\widehat{Z}_{\mathrm{sc}}$. It follows that $\lambda \widetilde{s}_{1} \lambda^{-1}=\widetilde{s}_{1}$. Suppose next that $\widetilde{s}_{2}$ belongs to the connected group $S_{\phi, \text { sc }}^{0}$. Then $\lambda \widetilde{s}_{2} \lambda^{-1}$ equals $\widetilde{s}_{2} z\left(\widetilde{s}_{2}, \lambda\right)$, for a 
point $z\left(\widetilde{s}_{2}, \lambda\right)$ in $\widehat{Z}_{\mathrm{sc}}^{\Gamma}$. The identity $\lambda \widetilde{s}_{2} \lambda^{-1}=\widetilde{s}_{2}$ follows from the obvious argument of deformation that takes $\widetilde{s}_{2}$ to the identity in $S_{\phi, \mathrm{sc}}^{0}$. If $z$ belongs to $\widehat{Z}_{\phi, \mathrm{sc} \text {,der, we }}$ combine the two identities to conclude that $\lambda z \lambda^{-1}=z$ for any $\lambda \in \phi\left(L_{F}\right)$, and hence that $z$ is $\Gamma$-invariant. The kernel $\widehat{Z}_{\phi \text {,sc,der }}$ is therefore contained in $\widehat{Z}_{\mathrm{sc}}^{\Gamma}$, as claimed.

We can form three chains of subgroups

$$
\begin{aligned}
\widehat{Z}_{\mathrm{sc}}^{0} \subset \widehat{Z}_{\phi, \mathrm{sc}, \mathrm{der}} & \subset \widehat{Z}_{\mathrm{sc}}^{\Gamma} \subset \widehat{Z}_{\mathrm{sc}} \subset S_{\phi, \mathrm{sc}}, \\
\widetilde{\mathcal{Z}}_{\phi, \text { der }} & \subset \widetilde{\mathcal{Z}}_{\phi}^{\Gamma} \subset \widetilde{\mathcal{Z}}_{\phi} \subset \widetilde{\mathcal{S}}_{\phi},
\end{aligned}
$$

and

$$
\widetilde{\mathcal{Z}}_{\phi, \mathrm{ab}}^{\Gamma} \subset \widetilde{\mathcal{Z}}_{\phi, \mathrm{ab}} \subset \widetilde{\mathcal{S}}_{\phi, \mathrm{ab}} .
$$

The groups in the upper chain have already been defined. The three left hand groups in the middle chain are defined as quotients by $\widehat{Z}_{\mathrm{sc}}^{0}$ of the corresponding groups in the upper chain. The two left hand groups in the lower chain are quotients by $\widetilde{\mathcal{Z}}_{\phi, \text { der }}$ of corresponding groups in the middle chain. In particular, the groups

and

$$
\widetilde{\mathcal{Z}}_{\phi, \mathrm{ab}}^{\Gamma}=\widetilde{\mathcal{Z}}_{\phi}^{\Gamma} / \widetilde{\mathcal{Z}}_{\phi, \mathrm{der}} \cong \widehat{Z}_{\mathrm{sc}}^{\Gamma} / \widehat{Z}_{\phi, \mathrm{sc}, \mathrm{der}}
$$

$$
\widetilde{\mathcal{Z}}_{\phi, \mathrm{ab}}=\widetilde{\mathcal{Z}}_{\phi} / \widetilde{\mathcal{Z}}_{\phi, \mathrm{der}} \cong \widehat{Z}_{\mathrm{sc}} / \widehat{Z}_{\phi, \mathrm{sc}, \mathrm{der}}
$$

are the images of $\widehat{Z}_{\mathrm{sc}}^{\Gamma}$ and $\widehat{Z}_{\mathrm{sc}}$ respectively in $\widetilde{\mathcal{S}}_{\phi, \mathrm{ab}}$. (Observe that the notation for the groups that are subquotients of $\widehat{Z}_{\mathrm{sc}}$ is inherited from $S_{\phi, \mathrm{sc}}$ and $\widetilde{\mathcal{S}}_{\phi}$, rather than being intrinsic to the groups themselves. In particular, $\widetilde{\mathcal{Z}}_{\phi, \text { der }}$ is not the derived group of $\widetilde{\mathcal{Z}}_{\phi}, \widetilde{\mathcal{Z}}_{\phi}^{\Gamma}$ is not the group of $\Gamma$-invariants in $\widetilde{\mathcal{Z}}_{\phi}$, and $\widetilde{\mathcal{Z}}_{\phi, \text { ab }}$ is not the abelianization of $\widetilde{\mathcal{Z}}_{\varphi}$.)

The canonical mappings

$$
\widehat{Z}_{\mathrm{sc}} / \widehat{Z}_{\mathrm{sc}}^{\Gamma} \longrightarrow \widetilde{\mathcal{Z}}_{\phi} / \widetilde{\mathcal{Z}}_{\phi}^{\Gamma} \longrightarrow \widetilde{\mathcal{Z}}_{\phi, \mathrm{ab}} / \widetilde{\mathcal{Z}}_{\phi, \mathrm{ab}}^{\Gamma}
$$

are isomorphisms. Any extension of the character $\widehat{\zeta}_{G}$ from $\widehat{Z}_{\mathrm{sc}}^{\Gamma}$ to $\widehat{Z}_{\mathrm{sc}}$ is therefore uniquely determined up to multiplication by a character on the finite abelian group $\widetilde{\mathcal{Z}}_{\phi, \mathrm{ab}} / \widetilde{\mathcal{Z}}_{\phi, \mathrm{ab}}^{\Gamma}$. But a character on $\widetilde{\mathcal{Z}}_{\phi, \mathrm{ab}} / \widetilde{\mathcal{Z}}_{\phi, \mathrm{ab}}^{\Gamma}$ can be extended to a character $\xi$ on $\widetilde{\mathcal{S}}_{\phi, \mathrm{ab}} / \widetilde{\mathcal{Z}}_{\phi, \mathrm{ab}}^{\Gamma}$, which is uniquely determined up to multiplication by a character on the quotient

$$
\widetilde{\mathcal{S}}_{\phi, \mathrm{ab}} / \widetilde{\mathcal{Z}}_{\phi, \mathrm{ab}} \cong \mathcal{S}_{\phi, \mathrm{ab}} .
$$

We can of course treat $\xi$ as a character on $\widetilde{\mathcal{S}}_{\phi} / \widetilde{\mathcal{Z}}_{\phi}^{\Gamma}$, which is uniquely determined up to multiplication by a character on the quotient

$$
\widetilde{\mathcal{S}}_{\phi} / \widetilde{\mathcal{Z}}_{\phi} \cong \mathcal{S}_{\phi}
$$


If $\rho(\Delta, \widetilde{s})$ satisfies the conditions of the conjecture, so does the product

$$
\left(\rho \xi^{-1}\right)(\Delta, \widetilde{s})=\rho(\Delta, \widetilde{s}) \xi(\widetilde{s})^{-1} .
$$

We can therefore arrange that the $\widehat{Z}_{\mathrm{sc}}$-central character $\widehat{\zeta}_{\rho}$ of $\rho$ be any extension of $\widehat{\zeta}_{G}$ to $\widehat{Z}_{\text {sc }}$, simply by multiplying $\rho$ by a character on $\widetilde{\mathcal{S}}_{\phi} / \widetilde{\mathcal{Z}}_{\phi}^{\Gamma}$. The role of the extension of $\widehat{\zeta}_{G}$ to $\widehat{Z}_{\mathrm{sc}}$ in the conjecture is therefore insignificant.

Of course, we could also modify $\rho$ by multiplication with a more general character on $\widetilde{\mathcal{S}}_{\phi}$. This has the effect of transforming the $\widehat{Z}_{\mathrm{sc}}$-central character $\widehat{\zeta}_{\rho}$ of $\rho$ to a character whose restriction to $\widehat{Z}_{\mathrm{sc}}^{\Gamma}$ differs from $\widehat{\zeta}_{G}$. We are assuming that $\phi$ is $G$-relevant. It is not difficult to establish from this condition that $\widehat{\zeta}_{G}$ is trivial on the subgroup $\widehat{Z}_{\mathrm{sc}}^{0}$ of $\widehat{Z}_{\mathrm{sc}}^{\Gamma}$, and therefore descends to a character on the quotient $\widetilde{\mathcal{Z}}_{\phi}^{\Gamma}$ of $\widehat{Z}_{\mathrm{sc}}^{\Gamma}$. If $\widehat{\zeta}_{G}$ is trivial on the larger subgroup $\widehat{Z}_{\phi, \mathrm{sc}, \mathrm{der}}$, it can be regarded as a character on $\widetilde{\mathcal{Z}}_{\phi}^{\Gamma}$ that is trivial on the subgroup $\widetilde{\mathcal{Z}}_{\phi \text {,der }}$, and hence as a character on the quotient $\widetilde{\mathcal{Z}}_{\phi, \text { ab }}^{\Gamma}$ of $\widetilde{\mathcal{Z}}_{\phi}^{\Gamma}$. In this case we can transform $\rho$ by a character on $\widetilde{\mathcal{S}}_{\phi}$ so that it lifts to a function on the quotient $\mathcal{S}_{\phi}$ of $\widetilde{\mathcal{S}}_{\phi}$. The hypothetical classification of $\Pi_{\phi}$ is then no different from that of the quasisplit case. On the other hand, if $\widehat{\zeta}_{G}$ is nontrivial on $\widehat{Z}_{\phi, \mathrm{sc} \text {,der, }}$, its restriction defines a nontrivial character on the subgroup $\widetilde{\mathcal{Z}}_{\phi, \text { der }}$ of $\widetilde{\mathcal{S}}_{\varphi, \text { der }}$. In this case, it cannot be removed by multiplying $\rho$ by a character in $\widetilde{\mathcal{S}}_{\phi}$. The hypothetical classification is then genuinely different from that of the quasisplit case.

In summary, the hypothetical objects of the conjecture are determined (at best) only up to translation by a character on $\widetilde{\mathcal{S}}_{\phi}$. If we allow the character to be nontrivial on the image of $\widehat{Z}_{\mathrm{sc}}$ in $\widetilde{\mathcal{S}}_{\phi}$, we can modify the extension $\widehat{\zeta}_{\rho}$ of $\widehat{\zeta}_{G}$ to $\widehat{Z}_{\text {sc }}$. If we also allow it to be nontrivial on the subgroup $\widehat{Z}_{\mathrm{sc}}^{\Gamma}$ of $\widehat{Z}_{\mathrm{sc}}$, we can sometimes even modify the restriction $\widehat{\zeta}_{G}$ of $\widehat{\zeta}_{\rho}$ to $\widehat{Z}_{\mathrm{sc}}^{\Gamma}$. However, since $\widehat{\zeta}_{G}$ is canonically attached to the inner twist $(G, \psi)$, we may as well consider only those characters of $\widetilde{\mathcal{S}}_{\phi}$ that are trivial on the image of $\widehat{Z}_{\mathrm{sc}}^{\Gamma}$.

Consider the assertion (b) of the conjecture. Suppose that $z \in \widehat{Z}_{\mathrm{sc}}$ stabilizes the conjugacy class in $\widetilde{\mathcal{S}}_{\varphi}$ of an element $\widetilde{s} \in S_{\phi, \text { sc }}$. Then

$$
z \widetilde{s}=\widetilde{s} z=\widetilde{t} \widetilde{s} \widetilde{t}^{-1} \widetilde{s}_{2}
$$

for elements $\widetilde{t} \in S_{\phi, \mathrm{sc}}$ and $\widetilde{s}_{2} \in S_{\phi, \mathrm{sc}}^{0}$. We can therefore write

$$
z=\widetilde{s}_{1} \widetilde{s}_{2}
$$

for elements $\widetilde{s}_{1}=\widetilde{t} \widetilde{s} \widetilde{t}^{-1} \widetilde{s}^{-1}$ and $\widetilde{s}_{2}=\widetilde{s} \widetilde{s}_{2}^{\prime} \widetilde{s}^{-1}$ in $S_{\phi, \mathrm{sc} \text {,der }}$ and $S_{\phi, \mathrm{sc}}^{0}$ respectively. It follows that $z$ belongs to $\widehat{Z}_{\phi, \mathrm{sc}, \mathrm{der}}$, and hence that $z$ lies in $\widehat{Z}_{\mathrm{sc}}^{\Gamma}$. The stabilizer in $\widehat{Z}_{\mathrm{sc}}$ of any conjugacy class in $\widetilde{\mathcal{S}}_{\phi}$ is thus contained in the subgroup $\widehat{Z}_{\mathrm{sc}}^{\Gamma}$ of $\widehat{Z}_{\mathrm{sc}}$. Since the restriction of $\widehat{\zeta}_{\rho}$ to $\widehat{Z}_{\mathrm{sc}}^{\Gamma}$ equals $\widehat{\zeta}_{G}$, it then follows that the space 
of $\widehat{\zeta}_{\rho}$-equivariant class functions on $\widetilde{\mathcal{S}}_{\phi}$ has dimension equal to the order of the set $\Gamma\left(\mathcal{S}_{\varphi}, \widehat{\zeta}_{G}\right)$. The number of irreducible characters $\theta_{\rho}$ on $\widetilde{\mathcal{S}}_{\phi}$ with $\widehat{Z}_{\mathrm{sc}}$-central character $\widehat{\zeta}_{\rho}$ is therefore equal to the order of $\Gamma\left(\mathcal{S}_{\varphi}, \widehat{\zeta}_{G}\right)$. Part (a) of the conjecture asserts that the set of such characters is bijective with the set $\Pi_{\varphi}$. The second part (b) of the conjecture is therefore implied by (a).

For purposes of illustration, suppose that the group $\mathcal{S}_{\varphi}$ is abelian. This is the case, for example, whenever $G$ is a classical group. The extension

$$
1 \longrightarrow \widetilde{\mathcal{Z}}_{\phi} \longrightarrow \widetilde{\mathcal{S}}_{\varphi} \longrightarrow \mathcal{S}_{\varphi} \longrightarrow 1
$$

is then metabelian (an extension of an abelian group by an abelian group), and any commutator in $\widetilde{\mathcal{S}}_{\varphi}$ lies in the normal subgroup $\widetilde{\mathcal{Z}}_{\phi}$. The derived group $\widetilde{\mathcal{S}}_{\varphi, \text { der }}$ of $\widetilde{\mathcal{S}}_{\varphi}$ therefore equals the subgroup $\widetilde{\mathcal{Z}}_{\varphi, \text { der }}$ of $\widetilde{\mathcal{Z}}_{\phi}^{\Gamma}$. Suppose that $\widetilde{s}$ belongs to $\widetilde{\mathcal{S}}_{\varphi}$. If $t$ belongs to $\mathcal{S}_{\phi}$, we write

$$
t \widetilde{s} t^{-1}=\widetilde{s} z(t)
$$

for a point $z(t)$ in $\widetilde{\mathcal{Z}}_{\phi \text {,der }}$. Now any element $s^{\prime} \in S_{\phi}$ that projects to the image $s$ of $\widetilde{s}$ in $\mathcal{S}_{\phi}$ determines a pair $\left(G^{\prime}, \phi^{\prime}\right)$ that maps to $(\phi, s)$. Suppose that $t^{\prime}$ is a representative of $t$ in $S_{\phi}$ such that the adjoint actions of $s^{\prime}$ and $t^{\prime}$ on the reductive group $S_{\phi}^{0}$ simultaneously preserve a splitting. It is then easy to see that the action of $\operatorname{Int}\left(t^{\prime}\right)$ on $\widehat{G}^{\prime}$ defines an element $\alpha(t)$ in $\operatorname{Out}_{G}\left(G^{\prime}\right)$. In fact, $\alpha(t)$ belongs to the subgroup $\operatorname{Out}_{G}\left(G^{\prime}, \phi^{\prime}\right)$ of elements in $\operatorname{Out}_{G}\left(G^{\prime}\right)$ that stabilize the $\widehat{G}^{\prime}$-orbit of the $L$-homomorphism $\phi^{\prime}$ from $L_{F}$ to $\mathcal{G}^{\prime}$. As $t^{\prime}$ varies, we obtain a surjective homomorphism

$$
t \longrightarrow \alpha(t)
$$

from $\mathcal{S}_{\varphi}$ onto $\operatorname{Out}_{G}\left(G^{\prime}, \phi^{\prime}\right)$ such that

$$
z(\alpha(t))=z(t)
$$

Thus, when $\mathcal{S}_{\phi}$ is abelian, the derived group $\widetilde{\mathcal{Z}}_{\phi \text {,der }}=\widetilde{\mathcal{S}}_{\phi \text {,der }}$ is generated by automorphisms of endoscopic data. We recall that it is this group that governs whether the conjectural structure of the packet $\Pi_{\varphi}$ will be materially different from that of the quasisplit case. The question is whether the character $\widehat{\zeta}_{G}$ on $\widetilde{\mathcal{Z}}_{\varphi}^{\Gamma}$ is nontrivial on the subgroup $\widetilde{\mathcal{Z}}_{\phi, \text { der }}$. The answer is therefore determined in this case by the known equivariance property (3.1) of the transfer mapping under automorphism.

Finally, let us consider a further special case. Suppose that $G$ is the subgroup of elements of norm 1 in a quaternion algebra over $F$. Then $G$ is an inner twist of the split group $S L(2)$, corresponding to the nontrivial character $\widehat{\zeta}_{G}$ on the group

$$
\widehat{Z}_{\mathrm{sc}}=\widehat{Z}_{\mathrm{sc}}^{\Gamma} \cong \mathbb{Z} / 2 \mathbb{Z}
$$


of order 2. The dual group of $G$ equals

$$
\widehat{G}=G L(2, \mathbb{C}) / \widehat{Z}
$$

where $\widehat{Z}$ is the center of $G L(2, \mathbb{C})$.

Suppose that $\phi$ is a Langlands parameter for $G$ whose image in $\widehat{G}$ is the abelian group

$$
S \cong \mathbb{Z} / 2 \mathbb{Z} \oplus \mathbb{Z} / 2 \mathbb{Z}
$$

generated by the images of elements $\left(\begin{array}{cc}1 & 0 \\ 0 & -1\end{array}\right)$ and $\left(\begin{array}{ll}0 & 1 \\ 1 & 0\end{array}\right)$. Then $\phi$ factors through a quotient of $L_{F}$ that corresponds to the Galois group of a biquadratic extension of $F$. The centralizer of $\phi\left(L_{F}\right)$ in $\widehat{G}$ again equals $S$. The parameter $\phi$ is therefore elliptic and $G$-relevant, and since $Z(\widehat{G})$ is trivial, the group $\mathcal{S}_{\phi}$ equals $S$. The preimage $\widetilde{\mathcal{S}}_{\varphi}$ of $S$ in $\widehat{G}_{\mathrm{sc}}=S L(2, \mathbb{C})$ is isomorphic to the quaternion group

$$
\{ \pm 1, \pm i, \pm j, \pm k\}
$$

of order 8. The subgroup $\widehat{Z}_{\mathrm{sc}}$ of $\widetilde{\mathcal{S}}_{\phi}$ equals both the center and the derived group of $\widetilde{\mathcal{S}}_{\varphi}$. The four characters of the group $\widetilde{\mathcal{S}}_{\varphi, \text { ab }}=\mathcal{S}_{\varphi}$ pull back to four onedimensional representations of $\widetilde{\mathcal{S}}_{\phi}$, with trivial central character. These account for the representations in the packet $\Pi_{\varphi^{*}}$, where $\varphi^{*}$ is the associated Langlands parameter for the group $G^{*}=S L(2)$. To see what remains, we note that the five sets

$$
\{1\},\{-1\},\{ \pm i\},\{ \pm j\},\{ \pm k\}
$$

are the conjugacy classes in $\widetilde{\mathcal{S}}_{\varphi}$. There is consequently one remaining irreducible character $\chi$ of $\widetilde{\mathcal{S}}_{\varphi}$. It has degree two and nontrivial central character. In particular, $\chi$ vanishes on the $\widehat{Z}_{\mathrm{sc}}$-stable conjugacy classes of $i, j$ and $k$. It follows that

$$
\chi(\widetilde{s})=\left\{\begin{aligned}
2, & \text { if } \widetilde{s}=1, \\
-2, & \text { if } \widetilde{s}=-1 \\
0, & \text { otherwise }
\end{aligned}\right.
$$

for any element $\widetilde{s} \in \widetilde{\mathcal{S}}_{\varphi}$. The conjecture therefore asserts that the packet $\Pi_{\varphi}$ consists of one representation $\pi$, with the property that for suitably normalized transfer factors $\Delta$,

$$
\Delta\left(\phi^{\prime}, \pi\right)= \begin{cases}2, & \text { if } G^{\prime}=G, \\ 0, & \text { otherwise }\end{cases}
$$

for any pair $\left(G^{\prime}, \phi^{\prime}\right)$ that maps to $\phi$. This is just what was established in [LL]. (See $[\mathrm{S} 1, \S 12(\mathrm{~B})]$.

We have concluded our brief observations on the conjectural parametrization of the packets $\Pi_{\phi}$. These remarks all pertain to elementary properties of the group $\mathcal{S}_{\varphi}$. They are intended to provide motivation for the conjecture, and perhaps 
also some circumstantial evidence. More serious support might be found in the orthogonality relations satisfied by the representations $\pi \in \Pi_{\phi}$, and properties of the numbers $\Delta\left(\phi^{\prime}, \pi\right)$ related to expected global multiplicity formulas. We shall not discuss such questions here. All being well, we shall take them up (in the context of classical groups) in [A3].

\section{REFERENCES}

[ABV] J. Adams, D. Barbasch, and D. Vogan, The Langlands Classification and Irreducible Characters for Real Reductive Groups, Progr. Math. 104, Birkhauser, Boston, 1992.

[AV] J. Adams and D. Vogan, L-groups, projective representations, and the Langlands classification, Amer. J. Math. 114 (1992), 45-138.

[A1] J. Arthur, A stable trace formula III. Proof of the main theorems, Annals of Math. 158 (2003), 769-873.

[A2] Singular invariant distributions and endoscopy, in preparation.

[A3] Automorphic Representations of Classical Groups, in preparation.

[B] A. Borel, Automorphic L-functions, in Automorphic Forms, Representations, and Lfunctions, Proc. Sympos. Pure Math. vol. 33, Part 2, Amer. Math. Soc., 1979, 27-61.

[HT] M. Harris and R. Taylor, On the Geometry and Cohomology of Some Simple Shimura Varieties, Ann. of Math. Studies 151, Princeton Univ. Press, Princeton, N.J., 2001.

$[\mathrm{H}] \quad$ G. Henniart, Une preuve simple des conjectures de Langlands de $G L(n)$ sur un corps p-adique, Invent. Math. 139 (2000), 439-455.

[KL] D. Kazhdan and G. Lusztig, Proof of the Deligne-Langlands conjecture for Hecke algebras, Invent. Math. 87 (1987), 153-215.

[KZ] A. Knapp and G. Zuckerman, Classification of irreducible tempered representations of semisimple groups, Ann. of Math. 116 (1982), 389-455.

[K] R. Kottwitz, Stable trace formula: elliptic singular terms, Math. Ann. 275 (1986), 365-399.

[LL] J.-P. Labesse and R. Langlands, L-indistinguishability for SL(2), Canad. J. Math. 31 (1979), 726-785.

[La1] R. Langlands, Les débuts d'une formule des traces stables, Publ. Math. Univ. Paris VII 13, 1983.

[La2] On the classification of irreducible representations of real algebraic groups, in Representation Theory and Harmonic Analysis on Semisimple Lie Groups, AMS Mathematical Surveys and Monographs, vol. 31, 1989, 101-170.

[LS] R. Langlands and D. Shelstad, On the definition of transfer factors, Math. Ann. 278 (1987), 219-271.

[LN] G. Laumon and B.C. Ngo, Le lemme fondamental pour les groupes unitaires, preprint, Université Paris 13, 2004.

[Lu] G. Lusztig, Classification of unipotent representations of simple p-adic groups, Int. Math. Res. Not. 11 (1995), 517-589.

[MW] C. Moeglin and J.-L. Waldspurger, Pacquets stables de représentations tempérés et de reduction unipotente pour $S O(2 n+1)$, Invent. Math. 152 (2003), 461-623.

[S1] D. Shelstad, Notes on L-indistinguishability (based on a lecture by R.P. Langlands), in Automorphic Forms, Representations and L-functions, Proc. Sympos. Pure Math. vol. 33, Part 2, Amer. Math. Soc., 1979, 193-204.

[S2] - L-indistinguishability for real groups, Math. Ann. 259 (1982), 385-430.

[T] J. Tate, Number theoretic background, in Automorphic Forms, Representations, and L-functions, Proc. Sympos. Pure Math. vol. 33, Part 2, Amer. Math. Soc., 1979, 3-26. 
[V] D. Vogan, The local Langlands conjecture, Contemp. Math. 145 (1993), 305-379.

[W1] J.-L. Waldspurger, Le lemme fondamental implique le transfer, Compositio Math. 105 (1997), 153-236.

[W2] Endoscopie et changement de caractéristique, preprint.

James Arthur

University of Toronto

Bahen Conthe, 6th Floor

40 St. George Street

Toronto, ON, M55 3E4

Canada

E-mail: arthur@math.toronto.edu 